\title{
Blood pressure risk factors in early adolescents: results from a Ugandan birth cohort
}

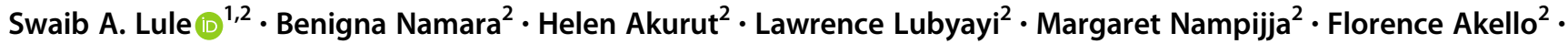 \\ Josephine Tumusiime ${ }^{2}$. Judith C. Aujo ${ }^{3}$. Gloria Oduru ${ }^{2}$ - Alexander J. Mentzer $\mathbb{B}^{4} \cdot$ Liam Smeeth $^{1}$. \\ Alison M. Elliott ${ }^{1,2} \cdot$ Emily L. Webb ${ }^{1}$
}

Received: 5 July 2018 / Revised: 25 January 2019 / Accepted: 11 February 2019 / Published online: 25 February 2019

(c) The Author(s) 2019. This article is published with open access

\begin{abstract}
We aimed to investigate life-course factors associated with blood pressure (BP) among Ugandan adolescents. Between 9th April 2003 and 24th November 2005, 2507 pregnant women from Entebbe municipality and Katabi sub-county were enrolled into a deworming trial. The resulting 2345 live-born offspring were followed to age 10 or 11 years, when between 20th May 2014 to 16th June 2016, BP was measured following standard protocols. Factors associated with BP were assessed using multivariable linear regression. BP was measured in 1119 adolescents with a median age of 10.2 years. Mean systolic $\mathrm{BP}$ and diastolic BP was $105.9 \mathrm{mmHg}$ (standard deviation (SD) 8.2) and $65.2 \mathrm{mmHg}$ (SD 7.3), respectively. Maternal gestational body mass index (BMI), higher maternal education status and family history of hypertension were positively associated with adolescent BP. Childhood (age $\leq 5$ years) malaria was associated with lower adolescent systolic BP. Factors measured at time of BP measurement positively associated with systolic BP were age, BMI, waist circumference and Trichuris trichiura (whipworm) infection; higher vegetable consumption was associated with lower systolic BP. Results for diastolic BP were similar, except higher fruit, rather than higher vegetable consumption was associated with lower diastolic BP and there was no association with waist circumference or Trichuris trichiura infection. In summary, life-course exposures were associated with adolescent BP in this tropical birth cohort. Malaria early in life could impact later BP. Interventions initiated early in life targeting individuals with family history of hypertension, aiming to reduce adiposity (in pregnancy and adolescence) and promoting fruit and vegetable consumption might contribute to reducing the risk of high BP and subsequent cardiovascular diseases.
\end{abstract}

Supplementary information The online version of this article (https:// doi.org/10.1038/s41371-019-0178-y) contains supplementary material, which is available to authorized users.

Swaib A. Lule

swaiblule@yahoo.com

1 London School of Hygiene and Tropical Medicine, Keppel Street, London WC1E 7HT, UK

2 MRC/UVRI \& LSHTM Uganda Research Unit, P.O. Box 49 Entebbe, Uganda

3 Department of Paediatrics, Mulago Hospital, P.O. Box 7051 Kampala, Uganda

4 Wellcome Trust Centre for Human Genetics, University of Oxford, Oxford, UK

\section{Introduction}

Once uncommon in Africa [1], high blood pressure (BP) and cardiovascular diseases (CVDs) have escalated on the continent over recent decades [2], affecting populations at younger ages than in more affluent countries [3], The rising burden of high BP in Africa has been attributed to a transition from active to more sedentary lifestyles and a rise in unhealthy dietary practices [2]. Data on individual level BP risk factors in African adolescents and children are sparse.

Although high BP is less common in children and adolescents than in adults, it initiates early in life, persists into adulthood [4] and predicts adulthood hypertension [5]. Diagnosis of CVDs is uncommon until middle-age, yet its antecedents, mainly cardiovascular and metabolic changes, begin early in life [6]. Globally, the high BP burden in younger age groups has risen [7], with estimated prevalence of $1-25 \%$ among African children and adolescents [8]. 
Severe persistent high BP is associated with increased risk of stroke and heart failure [9]; treatment reduces longterm sequelae [9]. In children and adolescents, high BP is often asymptomatic and unnoticed, despite international recommendations for regular BP measurement from three years of age [10]. Hypertension diagnosis is commonly missed or inaccurately classified in children and adolescents [11]. Consequently, over $75 \%$ of high BP among children and adolescents remains undiagnosed worldwide [12].

Earlier studies, mainly in adults, have demonstrated the role of established risk factors for high BP such as obesity [13] and physical activity [14]. There is little literature on childhood and adolescent BP determinants from Africa; in particular the impact of childhood infections (of special importance in Africa) remains understudied and unknown.

Childhood and adolescence are opportune periods for high BP control or prevention before clinical manifestation of hypertension or related CVDs. Identification of lifecourse BP risk factors unique to Africa is needed for the development of appropriate BP control strategies. We used longitudinally collected data from the Entebbe Mother and Baby Study (EMaBS), a large tropical birth cohort, to describe factors associated with adolescent BP.

\section{Methods}

\section{Study design, setting and population}

This longitudinal observational study investigated perinatal and life-course factors associated with BP among adolescents born in Wakiso district, Uganda. The EMaBS was a randomised double-blind placebo-controlled factorial trial [ISRCTN32849447], designed to investigate effects of worms and their treatment in pregnancy and childhood on response to childhood vaccines and on infections [15].

The study was conducted in Entebbe municipality and Katabi sub-county (a peninsula on the northern shores of Lake Victoria). In 2003-2005, 2507 women attending Entebbe Hospital antenatal clinic, in their second or third trimester were invited, enrolled and randomised to receive albendazole $(400 \mathrm{mg})$ or placebo and praziquantel $(40 \mathrm{mg} /$ $\mathrm{kg}$ ) or placebo [15].

Data were collected prenatally from women and resulting 2345 live-born offspring followed from birth. As previously described [16], at 15 months offspring were randomised to receive quarterly single-dose albendazole or placebo up to age five years. Disease events were recorded at the study clinic annually and when the child reported to the clinic with an illness. Children continued under follow-up (seen at routine annual visits and when sick) after trial completion. Between 20th May 2014 and 16th June 2016, additional data, including BP measurements, anthropometry, puberty, physical activity and diet were collected from 10- and 11year-olds. Enrolment into the BP study was postponed for those with malaria (fever with malaria parasites) or other illness until they were well after being treated by the study team. Clinic based field workers conducted home visits and telephone calls to remind participants of their annual visit and also invite them to participate in the BP study. Participants who then attended their 10- or 11-year annual visit during the BP study period were then invited to enrol and take part in the BP study at that visit. Adolescents participated once, on their first 10 or 11-year annual visit occurring during the study period.

\section{Study procedures}

Birth weight was measured and recorded immediately after birth in Entebbe hospital or obtained from child health cards for deliveries conducted elsewhere [17]. Weight and height at 10/11 years were measured with scales (Seca, Hamburg, Germany) and stadiometers (Seca 213, Hamburg Germany), respectively. Waist circumference was measured to the nearest $0.1 \mathrm{~cm}$ using a Seca tape measure (Seca 201, Hamburg, Germany). Body mass index (BMI) was calculated as weight in kilograms $(\mathrm{kg})$ divided by height squared $\left(\mathrm{m}^{2}\right)$. Trained clinicians examined and performed Tanner staging as described elsewhere [18].

Whole-genome genotyping of 1391 EMaBS samples was conducted at the Wellcome Trust Sanger Institute using Illumina HumanOmni2.5M-8 ('octo') Beadchip arrays, version 1.1 (Illumina Inc., San Diego, USA). Sickle-cell trait was imputed using a merged 1000 Genomes and African-specific reference panel [19].

For participants taking part in the BP study from the 21st January 2015 to 23rd December 2015, extra data on fat mass (FM), fat-free mass (FFM) and total body water mass (TBW) were collected by trained nurses using a segmental body composition analyser machine (SBCAM) [TANITA BC-418, TANITA Corporation, Tokyo Japan]. Briefly, participants stood barefooted on the posterior electrode base while holding two anterior electrodes handles of the SBCAM. Fat mass index $=$ FMI $(\mathrm{kg}) /$ height $\left(\mathrm{m}^{2}\right)$, fat-free mass index $=$ FFMI $(\mathrm{kg}) /$ height $\left(\mathrm{m}^{2}\right)$ and total body water mass index $=$ TBWI $(\mathrm{kg}) /$ height $\left(\mathrm{m}^{2}\right)$ were computed .

Stool and blood samples were collected from women at enrolment and annually from children. Stool was examined for helminth (Schistosoma mansoni, Necator americanus, Ascaris lumbricoides and Trichuris trichiura) ova and Strongyloides larvae using Kato-Katz [20] and charcoal culture [21] methods, respectively. Blood was examined for malaria parasites using Leishman's stains [16]. Modified Knott's method [22] was used for Mansonella perstans. Maternal HIV status at enrolment and children's HIV status after 18 months of age were assessed using a rapid serial 
testing algorithm described elsewhere [21, 23]. In infancy, HIV status was determined using polymerase chain reaction [21].

At the 10- or 11-year annual visit, three BP measurements (at $\sim 5$ min intervals) were taken after 5 min rest using automated devices (Omron M6), with appropriate sized cuffs [5], by trained nurses following standard protocols described elsewhere [17].

For clinical care purposes, means of the three systolic BP and three diastolic BP measurements were calculated and BP percentiles determined using Centre for Disease Control height charts and 2004 updated National Health and Nutrition Examination Survey BP tables specific for sex, age and height $[5,10]$. Those with mean systolic BP or diastolic BP $\geq 95$ th percentile ("high BP") had their BP remeasured on up to two extra days, 1-2 weeks apart. "Prehypertension" was defined as systolic or diastolic BP $\geq 90$ th but $<95$ th percentile. Those with persistent high BP on three different days were referred for specialist attention. Lifestyle modification was recommended for participants with systolic or diastolic BP $\geq 90$ th percentile.

For data analysis purposes, the means of the second and third systolic/diastolic BP readings on day 1 were used: day 1 second and third BP readings were lower than the first BP reading but similar to each other [17].

Ethical approval was granted by the Uganda Virus Research Institute Science and Ethics Committee; the Uganda National Council for Science and Technology; and the London School of Hygiene and Tropical Medicine. Written informed assent and consent were obtained for study participation.

\section{Statistical methods}

Data were collected on pre-coded questionnaires and analysed with Stata 14.2 (College Station, TX, USA). Chisquared tests (for categorical variables) and $t$-tests (for continuous variables) were used to compare characteristics of cohort members who participated and did not participate in the BP study.

Study outcomes were mean systolic BP and mean diastolic BP, based on the second and third day-one measurements. The decision was made to model these two continuous BP outcome variables rather than to dichotomise outcomes (for example, into normal versus hypertensive) as an analysis using these binary outcomes would be underpowered. Maternal, perinatal and offspring lifecourse factors considered as exposures and potential confounders were: maternal and adolescent sociodemographic and anthropometric characteristics; EMaBS trial interventions (praziquantel or albendazole); sicklecell trait; illnesses and infections from birth to time of BP measurement; and body composition, puberty stage, diet, sleep pattern and physical activity at time of BP measurement. Area of residence was grouped into urban versus rural area using zones based on topography and settlements generated from geographical positioning system data [24]. Household socioeconomic index was generated using principal components analysis of building materials, household size and items owned [23]. Birth season was dichotomised into dry (rainfall below monthly median) and wet (rainfall above monthly median) season. Malaria infection in childhood (age $\leq 5$ years) was investigated as clinical malaria (history of fever within the last $48 \mathrm{~h}$ or axillary temperature $\geq 37.5^{\circ} \mathrm{C}$ and parasitaemia) and asymptomatic malaria (parasitaemia without fever at any annual visit up to 5 years). Information on diet was obtained as the number of days in a typical week over the previous month for which a given food was consumed. Puberty was grouped into pre-pubertal (stage 1) or pubertal (stages 2-5) for breast or pubic hair development using Tanner methods [18].

Linear regression analysis was used. Data satisfied the assumptions for linear regression. Crude associations were examined for each covariate and a $20 \%$ significance level was used for selecting covariates for multivariable models. Adolescents' sex, age and BMI were confounders a priori. Multivariable analysis followed a hierarchical causal approach adding factors sequentially (Fig. 1).

Because of a large proportion of missing data, puberty and body composition variables were not included in model building for other exposures but their effects were each adjusted for variables included in the final multivariable model. Multicollinearity was assessed by considering the change in standard error, when potentially multicollinear variables were included in the same model.

\section{Results}

\section{Participant characteristics}

A total of 1119 EMaBS participants were enrolled into the BP study: 583 (52\%) were males; 1100 (98.3\%) singletons; $18(2 \%)$ HIV positive; and $344(31 \%)$ mixed feeding by 6 weeks. EMaBS adolescents participating in the BP study were similar to non-participants, except that mothers of participants were more likely to be of higher education status or married/cohabiting; offspring were less likely to be HIV positive or of a multiple birth, details published earlier [17].

At age 10/11 (median participant age 10.2 years (interquartile range (IQR): 10.0-10.9)), 117 (11\%) were attending boarding schools, $441(72 \%)$ were pre-pubertal stage for pubic hair development and $178(65 \%)$ of girls were prepubertal stage for breast development. Mean BMI was 
Fig. 1 Conceptual framework

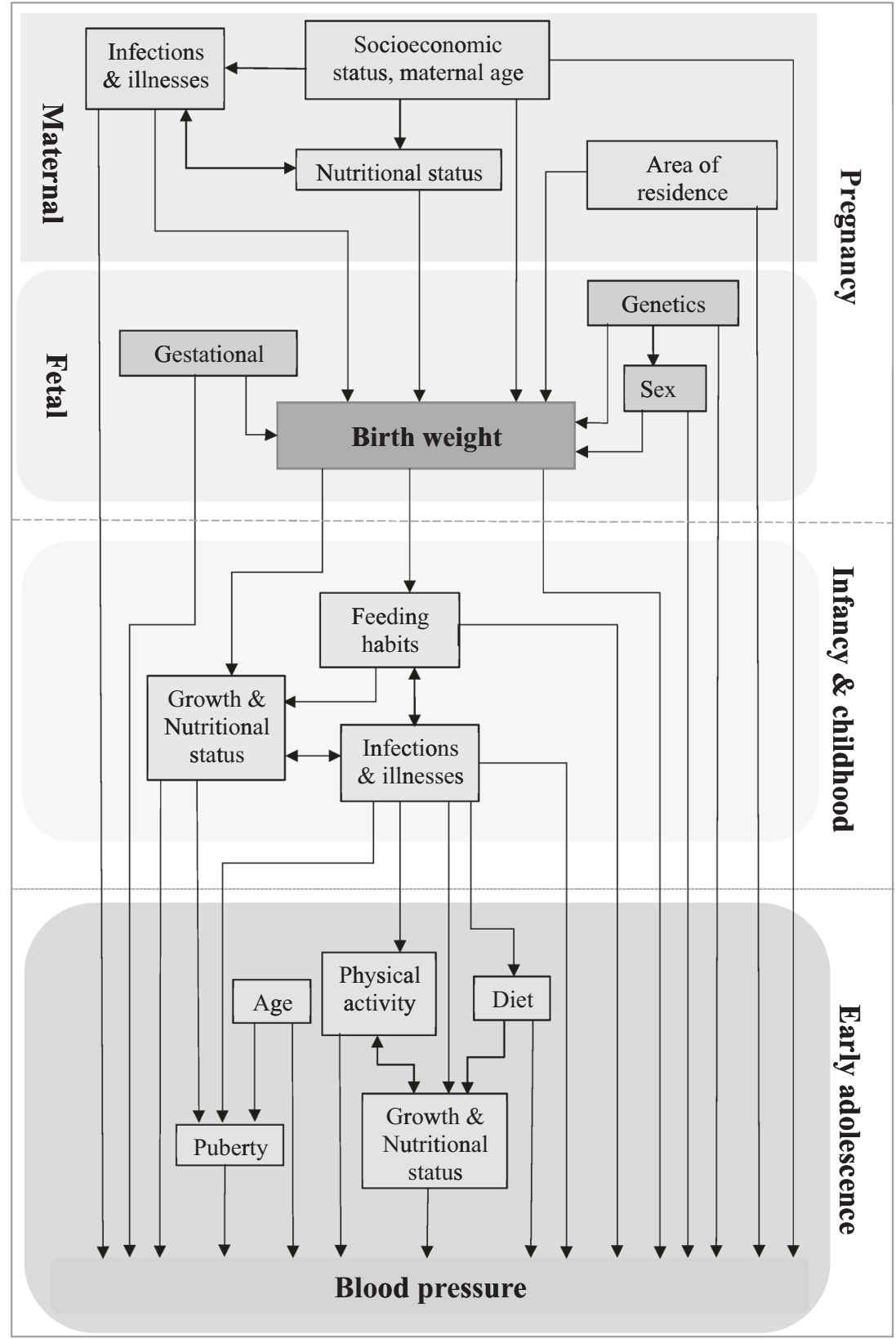

$15.8 \mathrm{~kg} / \mathrm{m}^{2}$ (standard deviation (SD) 1.9) and mean waist circumference $58.1 \mathrm{~cm}$ (SD 4.9). Body composition data were available for $176(16 \%)$ participants, with mean fat mass index $2.9 \mathrm{~kg} / \mathrm{m}^{2}$ (SD 1.2), fat-free mass index $12.8 \mathrm{~kg} /$ $\mathrm{m}^{2}$ (SD 1.4) and total body water mass index $9.5 \mathrm{~kg} / \mathrm{m}^{2}$ (SD 0.9).

Over the previous month, starchy staple foods, animal proteins, fruit, vegetables and sugar drinks were consumed on average for 6.9 days/week (SD 0.8), 2.2 days/ week (SD 1.7), 3 days/week (SD 2.2), 3.4 days/week (SD 2.3) and 1.7 days/week (SD 2.1), respectively. Nearly all adolescents (98\%) reported adding salt to cooked food.
Mean systolic BP was $105.9 \mathrm{mmHg}$ (SD 8.2) and mean diastolic BP was $65.2 \mathrm{mmHg}$ (SD 7.3). There was no difference in mean systolic BP $(P$-value $=0.971)$ or diastolic BP $(P$-value $=0.141)$ between males and females. None of the adolescents had had a prior BP measurement or high BP diagnosis.

\section{Prevalence of high blood pressure}

Using day 1 BP readings, the prevalence of prehypertension and high BP was $63(10.8 \%)$ and 42 (7.2\%), respectively, among males, and $54(10.1 \%)$ and $52(9.7 \%)$, respectively, among females. After extra measurements on 
the second and third visits and taking loss to follow-up into account, pre-hypertension prevalence was estimated as $2.2 \%$ in males and $0.7 \%$ in females; high BP prevalence was $0.4 \%$ in males and $1.8 \%$ in females.

\section{Risk factors for high blood pressure}

Tables 1 and 2 show the relationship between examined characteristics and BP (systolic or diastolic) in adolescents. Maternal factors crudely positively associated with adolescent systolic BP were gestational BMI and education status; both remained associated with systolic BP after adjustment for other maternal factors. The trial interventions during pregnancy (albendazole and praziquantel) and early childhood (albendazole) had no effect on systolic or diastolic BP.

Characteristics at the time of BP measurement showing a crude positive association with systolic BP were age, BMI, waist circumference, family history of high BP, body composition variables and puberty stage covariates. In multivariable analysis, systolic BP increased, on average, by $1.35 \mathrm{mmHg}, 95 \% \mathrm{CI}(0.32,2.39)$ for each 1 -year increase in adolescents' age; by $0.78 \mathrm{mmHg}(0.42,1.14)$ per unit increase in BMI; and by $0.21 \mathrm{mmHg}(0.08,0.35)$ per centimetre increase in waist circumference. Family history of high BP remained associated with increased systolic BP, $\beta=1.84(0.12,3.56)$ after adjustment for maternal and childhood factors. Body composition and puberty stage covariates were no longer associated with systolic BP on adjusting for adolescents' age, BMI and waist circumference.

Lifestyle factors crudely associated with increased systolic BP were increased animal-protein consumption, increased consumption of sugared drinks and attending a boarding school rather than a day school. Increased fruit and vegetable consumption were associated with reduced systolic BP. After adjusting for confounders, systolic BP reduced on average by $1.13 \mathrm{mmHg}(-2.15,-0.10)$ among adolescents who consumed vegetables for 3-7 days/week (versus 0-2 days/week).

Current infection with Trichuris trichiura was positively associated with systolic BP after adjusting for confounders $(\beta=3.48 \mathrm{mmHg}(0.79,6.18))$. Systolic BP dropped by $1.24 \mathrm{mmHg}(-2.32,-0.17)$ among adolescents who had malaria in childhood compared to those who had not. Both clinical and asymptomatic malaria were independently associated with lower BP in multivariable analysis. Weight and height at 10 and 11 years of age were reduced among adolescents with childhood clinical and or asymptomatic malaria (Supplementary Table 1). Compared to those with no asymptomatic malaria, having asymptomatic malaria in childhood was associated with, on average, a $3.2 \mathrm{~cm}$ reduction in height, 95\% CI $(-4.5,-2.0)$ and a $2.1 \mathrm{~kg}$ reduction in weight, $95 \% \mathrm{CI}(-3.0,-1.9)$. The effect of childhood malaria on adolescent BP was weaker on adjusting for adolescent BMI (Supplementary Table 2).

Genetic data were available for $802(72 \%)$ participants of whom 141 (18\%) had sickle-cell trait (HbAS) and 661 $(82 \%)$ normal haemoglobin (HbAA). Sickle-cell trait was not associated with systolic BP $(\beta=-0.28 \mathrm{mmHg}(-1.79$, $1.23)$ ), even after adjusting for age and sex. HbAS was inversely associated with malaria (Supplementary Table 3): in those with $\mathrm{HbAA}, 63 \%$ had clinical or asymptomatic malaria up to 5 years compared to $51 \%$ with $\mathrm{HbAS}(P$ value $=0.008$ )

Findings for diastolic BP were broadly similar to those for systolic BP, with the exceptions that higher fruit rather than vegetable consumption was associated with lower diastolic BP, and there was no association with waist circumference or Trichuris infection. No associations were observed between adolescent BP and any of the other factors considered in this population (Tables 1 and 2).

\section{Discussion}

Persistent high BP and pre-hypertension were unusual in early adolescence in this setting. Maternal gestational BMI and education status at enrolment, participant's family history of hypertension, and adolescents' age and BMI at BP measurement were positively associated with both systolic BP and diastolic BP. Malaria parasitaemia in childhood, and increased vegetable and fruit consumption were inversely associated with systolic BP and diastolic BP, respectively. Concurrent Trichuris infection was positively associated with systolic BP but not with diastolic BP. There were no effects of anti-helminth trial interventions (in pregnancy or childhood) on adolescent BP and no associations between prior helminth infection (in pregnancy or childhood) and adolescent BP.

Our findings are consistent with several earlier studies $[25,26]$. We have shown that consuming vegetables and fruits for 3-7 days/week was associated with lower systolic $\mathrm{BP}$ and diastolic BP, respectively. Our results support findings from a cross-sectional study that consuming fruits and vegetables (>400 g/day) lowers systolic BP and diastolic BP in adults [26]. We have shown a positive association of BP with maternal gestational BMI, and adolescent BMI and waist circumference at the time of BP measurement, consistent with earlier studies [13].

Malaria parasitaemia in childhood was associated with lower BP in early adolescence, consistent with findings from a cross-sectional study among 5-18-year-old Ugandan students, which reported that current asymptomatic malaria was associated with lower BP [25]. Our study was underpowered to detect the effect of current parasitaemia on BP, 
Table 1 Factors investigated for association with systolic blood pressure among adolescents from the Entebbe Mother and Baby Study $(N=1119)$

\begin{tabular}{|c|c|c|c|c|c|}
\hline Factors & Mean BP (SD) & Crude $\beta(95 \% \mathrm{CI})$ & $P$-value & Adjusted $\beta(95 \% \mathrm{CI})$ & $P$-value \\
\hline \multicolumn{6}{|l|}{ Level 1: Maternal factors at enrolments } \\
\hline Age (years) & & $0.06(-0.03,0.15)$ & 0.178 & $0.02(-0.07,0.12)$ & 0.604 \\
\hline $\begin{array}{l}\text { Household socioeconomic index } \\
(n=1104)\end{array}$ & & $0.23(-0.16,0.63)$ & 0.245 & & \\
\hline Parity & & $0.04(-0.23,0.31)$ & 0.751 & & \\
\hline $\begin{array}{l}\text { Body mass index }\left(\mathrm{kg} / \mathrm{m}^{2}\right) \\
(n=1110)\end{array}$ & & $0.27(0.13,0.42)$ & $<0.001$ & $0.26(0.11,0.40)$ & $<0.001$ \\
\hline \multicolumn{6}{|l|}{ Education status } \\
\hline None $(n=28)$ & $104.5(8.7)$ & $-0.54(-3.65,2.56)$ & & $-0.62(-3.77,2.53)$ & \\
\hline Primary $(n=542)$ & $105.0(7.7)$ & Reference & & Reference & \\
\hline Senior $(n=438)$ & $106.5(8.2)$ & $1.45(0.42,2.48)$ & & $1.43(0.39,2.47)$ & \\
\hline Tertiary $(n=109)$ & $108.2(9.8)$ & $3.19(1.51,4.87)$ & $<0.001$ & $3.14(1.45,4.84)$ & $<0.001$ \\
\hline \multicolumn{6}{|l|}{ Marital status } \\
\hline Single $(n=116)$ & $104.7(7.6)$ & $-1.34(-2.92,0.25)$ & & & \\
\hline Married/cohabiting $(n=967)$ & $106.0(8.3)$ & Reference & & & \\
\hline Separated/widowed $(n=35)$ & $105.3(6.1)$ & $-0.78(-3.56,1.99)$ & 0.229 & & \\
\hline \multicolumn{6}{|l|}{ Area of residence } \\
\hline Urban $(n=770)$ & $106.0(8.3)$ & Reference & & & \\
\hline Rural $(n=336)$ & $105.5(8.0)$ & $-0.47(-1.52,0.59)$ & 0.386 & & \\
\hline \multicolumn{6}{|l|}{ Alcohol use } \\
\hline No $(n=775)$ & $105.8(8.4)$ & Reference & & & \\
\hline Yes $(n=343)$ & $106.0(7.8)$ & $0.15(-0.90,1.19)$ & 0.781 & & \\
\hline \multicolumn{6}{|l|}{ Infections } \\
\hline \multicolumn{6}{|l|}{ HIV } \\
\hline Uninfected $(n=1002)$ & $106.0(8.3)$ & Reference & & Reference & \\
\hline Infected $(n=117)$ & $104.8(7.2)$ & $-1.17(-2.74,0.41)$ & 0.146 & $-0.88(-2.48,0.72)$ & 0.279 \\
\hline \multicolumn{6}{|l|}{ Asymptomatic malaria } \\
\hline Uninfected $(n=991)$ & $105.8(8.2)$ & Reference & & & \\
\hline Infected $(n=109)$ & $106.2(8.6)$ & $0.42(-1.20,2.05)$ & 0.609 & & \\
\hline \multicolumn{6}{|l|}{ Schistosoma mansoni } \\
\hline Uninfected $(n=908)$ & $105.8(8.3)$ & Reference & & & \\
\hline Infected $(n=204)$ & $106.2(7.9)$ & $0.35(-0.90,1.61)$ & 0.578 & & \\
\hline \multicolumn{6}{|l|}{ Hookworm (Necator americanus) } \\
\hline Uninfected $(n=662)$ & $105.8(8.1)$ & Reference & & & \\
\hline Infected $(n=450)$ & $105.9(8.4)$ & $0.10(-0.89,1.09)$ & 0.844 & & \\
\hline \multicolumn{6}{|l|}{ Ascaris lumbricoides } \\
\hline Uninfected $(n=1084)$ & $105.9(8.3)$ & Reference & & & \\
\hline Infected $(n=28)$ & $105.7(6.7)$ & $-0.17(-3.27,2.92)$ & 0.912 & & \\
\hline \multicolumn{6}{|l|}{ Intervention one } \\
\hline Placebo $(n=566)$ & $105.5(8.2)$ & Reference & & Reference & \\
\hline Albendazole $(n=553)$ & $106.2(8.3)$ & $0.67(-0.29,1.63)$ & 0.173 & $0.84(-0.12,1.80)$ & 0.087 \\
\hline \multicolumn{6}{|l|}{ Intervention two } \\
\hline Placebo $(n=564)$ & $106.0(8.1)$ & Reference & & & \\
\hline Praziquantel $(n=555)$ & $105.8(8.4)$ & $-0.20(-1.16,0.77)$ & 0.686 & & \\
\hline \multicolumn{6}{|l|}{ Level 2: Factors in childhood } \\
\hline Birth weight $(\mathrm{kg})(n=932)$ & & $0.73(-0.33,1.80)$ & 0.178 & $0.18(-0.93,1.29)$ & 0.751 \\
\hline Sex & & & & & \\
\hline Male $(n=583)$ & $105.9(7.5)$ & Reference & & Reference & \\
\hline Female $(n=536)$ & $105.9(9.0)$ & $-0.02(-0.98,0.95)$ & 0.971 & $0.12(-1.18,0.94)$ & 0.819 \\
\hline Sickle-cell trait & & & & & \\
\hline $\operatorname{HbAA}(n=661)$ & $106.0(8.4)$ & Reference & & & \\
\hline HbAS $(n=141)$ & $105.8(7.9)$ & $-0.28(-1.79,1.23)$ & 0.717 & & \\
\hline Season of birth & & & & & \\
\hline Dry $(n=651)$ & $106.1(8.1)$ & Reference & & & \\
\hline Wet $(n=468)$ & $105.5(8.3)$ & $-0.56(-1.54,0.42)$ & 0.261 & & \\
\hline Place of delivery & & & & & \\
\hline Entebbe Hospital $(n=824)$ & $105.8(8.2)$ & Reference & & Reference & \\
\hline Home $(n=120)$ & $104.9(8.6)$ & $-0.86(-2.43,0.71)$ & & $-0.37(-3.71,2.96)$ & \\
\hline Others $(n=174)$ & $106.8(8.0)$ & $0.95(-0.39,2.29)$ & 0.166 & $0.90(-0.87,2.68)$ & 0.582 \\
\hline Feeding status (at 6 weeks of age) & & & & & \\
\hline Exclusively breast fed $(n=748)$ & $106.1(8.2)$ & Reference & & & \\
\hline Mixed fed $(n=344)$ & $105.4(8.4)$ & $-0.70(-1.75,0.35)$ & & & \\
\hline Weaned $(n=14)$ & $105.8(7.1)$ & $-0.28(-4.63,4.08)$ & 0.430 & & \\
\hline Intervention three & & & & & \\
\hline Placebo $(n=553)$ & $105.5(8.4)$ & Reference & & & \\
\hline Albendazole $(n=554)$ & $106.1(8.0)$ & $0.61(-0.36,1.58)$ & 0.218 & & \\
\hline
\end{tabular}


Table 1 (continued)

\begin{tabular}{|c|c|c|c|c|c|}
\hline Factors & Mean BP (SD) & Crude $\beta(95 \% \mathrm{CI})$ & $P$-value & Adjusted $\beta(95 \% \mathrm{CI})$ & $P$-value \\
\hline \multicolumn{6}{|l|}{ HIV status } \\
\hline Unexposed $(n=1001)$ & $106.0(8.3)$ & Reference & & Reference & \\
\hline Exposed not infected $(n=100)$ & $105.2(7.3)$ & $-0.83(-2.52,0.86)$ & & $-0.29(-2.15,1.57)$ & \\
\hline Infected $(n=18)$ & $102.7(6.1)$ & $-3.34(-7.17,0.49)$ & 0.156 & $-3.85(-7.81,0.12)$ & 0.157 \\
\hline \multicolumn{6}{|l|}{ Malaria infection below 5 years of age } \\
\hline \multicolumn{6}{|l|}{ Clinical or asymptomatic ${ }^{\mathrm{a}}$} \\
\hline None $(n=456)$ & $106.6(8.0)$ & Reference & & Reference & \\
\hline Yes $(n=663)$ & $105.3(8.3)$ & $-1.31(-2.29,-0.33)$ & 0.009 & $-1.24(-2.32,-0.17)$ & 0.023 \\
\hline \multicolumn{6}{|l|}{ Clinical malaria ${ }^{\mathrm{a}}$} \\
\hline None $(n=474)$ & $106.6(8.0)$ & Reference & & Reference & \\
\hline Yes $(n=645)$ & $105.4(8.3)$ & $-1.19(-2.17,-0.22)$ & 0.016 & $-1.08(-2.15,-0.02)$ & 0.045 \\
\hline \multicolumn{6}{|l|}{ Episodes of clinical malaria ${ }^{a}$} \\
\hline None $(n=474)$ & $106.6(8.0)$ & Reference & & Reference & \\
\hline $1-2(n=382)$ & $105.4(8.4)$ & $-1.13(-2.24,-0.03)$ & & $-1.11(-2.32,0.11)$ & \\
\hline$\geq 3(n=263)$ & $105.3(8.2)$ & $-1.28(-2.52,-0.04)$ & 0.026 [trend] & $-1.05(-2.41,0.31)$ & 0.133 \\
\hline \multicolumn{6}{|l|}{ Asymptomatic malaria $^{a}$} \\
\hline None $(n=983)$ & $106.1(8.2)$ & Reference & & Reference & \\
\hline Yes $(n=124)$ & $103.7(8.0)$ & $-2.41(-3.94,-0.88)$ & 0.002 & $-1.95(-3.70,-0.20)$ & 0.028 \\
\hline \multicolumn{6}{|l|}{ Schistosoma mansoni } \\
\hline Uninfected $(n=1076)$ & $105.9(8.2)$ & Reference & & & \\
\hline Infected $(n=33)$ & $104.8(7.9)$ & $-1.09(-3.94,1.76)$ & 0.452 & & \\
\hline \multicolumn{6}{|l|}{ Ascaris lumbricoides } \\
\hline Uninfected $(n=1052)$ & $105.9(8.3)$ & Reference & & & \\
\hline Infected $(n=57)$ & $105.3(7.3)$ & $-0.62(-2.82,1.57)$ & 0.576 & & \\
\hline \multicolumn{6}{|l|}{ Hookworm (Necator americanus) } \\
\hline Uninfected $(n=1085)$ & $105.9(8.2)$ & Reference & & & \\
\hline Infected $(n=24)$ & $103.8(8.9)$ & $-2.06(-5.38,1.27)$ & 0.225 & & \\
\hline \multicolumn{6}{|l|}{ Trichuris trichiura } \\
\hline Uninfected $(n=997)$ & $105.9(8.2)$ & Reference & & & \\
\hline Infected $(n=112)$ & $105.6(8.6)$ & $-0.28(-1.89,1.33)$ & 0.731 & & \\
\hline \multicolumn{6}{|l|}{ Microfilaria (Mansonella perstans) } \\
\hline Uninfected $(n=1102)$ & $105.8(8.2)$ & Reference & & & \\
\hline Infected $(n=8)$ & $109.4(8.9)$ & $3.58(-2.13,9.28)$ & 0.219 & & \\
\hline \multicolumn{6}{|l|}{ Level 3: Factors in adolescence } \\
\hline Age (years) & & $2.12(1.17,3.08)$ & $<0.001$ & $1.35(0.32,2.39)$ & 0.009 \\
\hline Body mass index $\left(\mathrm{kg} / \mathrm{m}^{2}\right)$ & & $1.27(1.02,1.51)$ & $<0.001$ & $0.78(0.42,1.14)$ & $<0.001$ \\
\hline Waist circumference (cm) & & $0.46(0.36,0.55)$ & $<0.001$ & $0.21(0.08,0.35)$ & 0.002 \\
\hline \multicolumn{6}{|l|}{ Family history } \\
\hline High blood pressure & & & & & \\
\hline No $(n=1000)$ & $105.7(8.1)$ & Reference & & Reference & \\
\hline Yes $(n=105)$ & $107.6(8.3)$ & $1.88(0.24,3.52)$ & 0.025 & $1.84(0.12,3.56)$ & 0.034 \\
\hline Diabetes & & & & & \\
\hline No $(n=927)$ & $105.8(8.0)$ & Reference & & & \\
\hline Yes $(n=186)$ & $106.4(9.2)$ & $0.69(-0.61,1.99)$ & 0.296 & & \\
\hline Body composition analysis ${ }^{\mathrm{c}}$ & & & & & \\
\hline Fat mass index ${ }^{\mathrm{b}}\left(\mathrm{kg} / \mathrm{m}^{2}\right)(n=176)$ & & $3.27(2.29,4.24)$ & $<0.001$ & $1.50(-0.38,3.38)$ & 0.089 \\
\hline Fat-free mass index ${ }^{\mathrm{b}}\left(\mathrm{kg} / \mathrm{m}^{2}\right)(n=176)$ & & $1.54(0.65,2.43)$ & 0.001 & $-0.86(-2.25,0.54)$ & 0.188 \\
\hline Total body water index ${ }^{\mathrm{b}}\left(\mathrm{kg} / \mathrm{m}^{2}\right)(n=176)$ & & $4.20(2.97,5.42)$ & $<0.001$ & $2.51(-0.24,5.27)$ & 0.052 \\
\hline Adding salt to cooked food at the table & & & & & \\
\hline No $(n=20)$ & $106.2(7.3)$ & $0.36(-3.28,4.00)$ & & & \\
\hline Yes $(n=1086)$ & $105.9(8.2)$ & Reference & 0.846 & & \\
\hline Days a fruit is eaten/week & & & & & \\
\hline $0-2(n=543)$ & $106.3(8.0)$ & Reference & & Reference & \\
\hline $3-7(n=541)$ & $105.5(8.5)$ & $-0.83(-1.82,0.15)$ & 0.098 & $-0.83(-1.84,0.19)$ & 0.106 \\
\hline Days vegetables eaten/week & & & & & \\
\hline $0-2(n=461)$ & $106.4(8.2)$ & Reference & & Reference & \\
\hline $3-7(n=635)$ & $105.5(8.3)$ & $-0.94(-1.93,0.05)$ & 0.063 & $-1.13(-2.15,-0.10)$ & 0.029 \\
\hline Days animal-protein eaten/week & & & & & \\
\hline $0-2(n=726)$ & $105.4(7.8)$ & Reference & & Reference & \\
\hline $3-7(n=374)$ & $106.6(8.8)$ & $1.17(0.16,2.19)$ & 0.024 & $0.99(-0.06,2.04)$ & 0.062 \\
\hline Days sugared drinks taken/week & & & & & \\
\hline None $(n=427)$ & $105.4(8.1)$ & Reference & & Reference & \\
\hline $1-3(n=492)$ & $105.9(8.0)$ & $0.54(-0.53,1.61)$ & & $-0.05(-1.14,1.05)$ & \\
\hline $4-7(n=174)$ & $107.2(9.1)$ & $1.81(0.36,3.26)$ & 0.051 & $0.96(-0.53,2.44)$ & 0.358 \\
\hline Days a fruit is eaten/week & & $-0.05(-0.27,0.18)$ & 0.687 & & \\
\hline
\end{tabular}


Table 1 (continued)

\begin{tabular}{|c|c|c|c|c|c|}
\hline Factors & Mean BP (SD) & Crude $\beta(95 \% \mathrm{CI})$ & $P$-value & Adjusted $\beta(95 \% \mathrm{CI})$ & $P$-value \\
\hline Days vegetables eaten/week & & $-0.18(-0.39,0.03)$ & 0.085 & $-0.19(-0.40,0.03)$ & 0.081 \\
\hline Days animal-protein eaten/week & & $0.21(-0.07,0.50)$ & 0.138 & $0.10(-0.20,0.39)$ & 0.502 \\
\hline Days starchy foods eaten/week & & $0.14(-0.45,0.73)$ & 0.636 & & \\
\hline Days sugared drinks taken/week & & $0.23(0.00,0.46)$ & 0.049 & $0.11(-0.12,0.35)$ & 0.325 \\
\hline \multicolumn{6}{|l|}{ Breast development (girls only) ${ }^{\mathbf{b}}$} \\
\hline Pre-pubertal $(n=178)$ & $103.9(7.8)$ & Reference & & Reference & \\
\hline Pubertal $(n=97)$ & $108.0(10.5)$ & $4.07(1.87,6.26)$ & $<0.001$ & $1.17(-1.26,3.59)$ & 0.318 \\
\hline \multicolumn{6}{|l|}{ Pubic hair development ${ }^{\mathrm{b}}$} \\
\hline Pre-pubertal $(n=441)$ & $104.7(7.4)$ & Reference & & Reference & \\
\hline Pubertal $(n=170)$ & $106.5(9.3)$ & $1.83(0.42,3.24)$ & 0.011 & $0.51(-0.96,1.98)$ & 0.486 \\
\hline \multicolumn{6}{|l|}{ Snoring } \\
\hline No $(n=932)$ & $105.8(8.2)$ & Reference & & & \\
\hline Yes $(n=163)$ & $106.3(8.2)$ & $0.53(-0.83,1.90)$ & 0.444 & & \\
\hline \multicolumn{6}{|l|}{ Duration of night sleep } \\
\hline$<9$ hours $(n=306)$ & $106.1(8.0)$ & Reference & & & \\
\hline 9 hours $(n=382)$ & $105.8(8.8)$ & $-0.28(-1.51,0.96)$ & & & \\
\hline$>9$ hours $(n=405)$ & $105.7(7.7)$ & $-0.39(-1.61,0.83)$ & 0.818 & & \\
\hline \multicolumn{6}{|l|}{ Smoking in household } \\
\hline No $(n=962)$ & $106.0(8.3)$ & Reference & & Reference & \\
\hline Yes $(n=147)$ & $104.9(7.5)$ & $-1.03(-2.46,0.40)$ & 0.157 & $-0.65(-2.10,0.80)$ & 0.372 \\
\hline \multicolumn{6}{|l|}{ Type of school attended } \\
\hline Day $(n=117)$ & $105.7(7.9)$ & Reference & & Reference & \\
\hline Boarding school $(n=719)$ & $107.5(10.3)$ & $1.76(0.19,3.34)$ & 0.038 & $0.28(-1.38,1.95)$ & 0.733 \\
\hline \multicolumn{6}{|c|}{ Involved in physical education at school } \\
\hline No $(n=385)$ & $105.5(8.5)$ & Reference & & & \\
\hline Yes $(n=719)$ & $106.0(8.1)$ & $0.48(-0.54,1.50)$ & 0.360 & & \\
\hline \multicolumn{6}{|c|}{ Infections at the time of blood pressure measurement } \\
\hline \multicolumn{6}{|c|}{ Asymptomatic malaria } \\
\hline Uninfected $(n=1067)$ & $106.0(8.2)$ & Reference & & Reference & \\
\hline Infected $(n=22)$ & $103.1(9.3)$ & $-2.85(-6.31,0.61)$ & 0.106 & $-1.50(-5.02,2.02)$ & 0.397 \\
\hline \multicolumn{6}{|l|}{ Schistosoma mansoni } \\
\hline Uninfected $(n=964)$ & $105.9(8.3)$ & Reference & & & \\
\hline Infected $(n=112)$ & $105.7(8.4)$ & $-0.25(1.88,1.38)$ & 0.764 & & \\
\hline \multicolumn{6}{|c|}{ Hookworm (Necator americanus) } \\
\hline Uninfected $(n=1066)$ & $105.9(8.3)$ & Reference & & & \\
\hline Infected $(n=10)$ & $103.8(10.0)$ & $-2.10(-7.27,3.07)$ & 0.425 & & \\
\hline \multicolumn{6}{|l|}{ Ascaris (Ascaris lumbricoides) } \\
\hline Uninfected $(n=1073)$ & $105.9(8.3)$ & Reference & & Reference & \\
\hline Infected $(n=3)$ & 98.7 (1.6) & $-7.34(-16.65,2.17)$ & 0.132 & $-7.04(-15.97,1.88)$ & 0.117 \\
\hline \multicolumn{6}{|l|}{ Trichuris trichiura } \\
\hline Uninfected $(n=1036)$ & $105.8(8.3)$ & Reference & & Reference & \\
\hline Infected $(n=40)$ & $107.9(8.3)$ & $2.16(-0.46,4.78)$ & 0.106 & $3.48(0.79,6.18)$ & 0.010 \\
\hline
\end{tabular}

Model building followed the hierarchical approach, adding factors sequentially at three levels starting with the distal factors (level 1). Factors at the same level were added to the model at the same time and considered confounders for each other and for proximal factors. A $P$-value $<0.20$ was used for considering the inclusions and maintenance of factors in the model

Adjusted $\beta$ with $95 \%$ CI excluding 0 in bold

$\beta$ linear regression coefficient: mean difference in blood pressure (BP) measured in $\mathrm{mmHg}$

${ }^{a}$ Not included in the model together but each was adjusted for all other model variables

${ }^{b}$ Not included in multivariable model building for other exposures because of large proportion of missing information but each was adjusted for variables in the final model building

${ }^{c}$ Not adjusted for body mass index because body mass index is on the causal pathway 
Table 2 Factors investigated for association with diastolic blood pressure among adolescents from the Entebbe Mother and Baby Study $(N=1119)$

\begin{tabular}{|c|c|c|c|c|c|}
\hline Factors & Mean BP (SD) & Crude $\beta(95 \% \mathrm{CI})$ & $P$-value & Adjusted $\beta(95 \% \mathrm{CI})$ & $P$-value \\
\hline \multicolumn{6}{|l|}{ Level 1: Maternal factors } \\
\hline Age (years) & & $0.08(-0.00,0.15)$ & 0.058 & $0.05(-0.03,0.13)$ & 0.247 \\
\hline Household socioeconomic index $(n=1104)$ & & $0.22(-0.13,0.56)$ & 0.225 & & \\
\hline Parity & & $0.08(-0.16,0.32)$ & 0.530 & & \\
\hline Body mass index $(n=1110)$ & & $0.16(0.03,0.29)$ & 0.014 & $0.14(0.01,0.27)$ & $\mathbf{0 . 0 3 0}$ \\
\hline \multicolumn{6}{|l|}{ Education status } \\
\hline None $(n=28)$ & $65.1(9.3)$ & $0.44(-2.32,3.19)$ & & $0.08(-2.71,2.89)$ & \\
\hline Primary $(n=542)$ & $64.6(6.7)$ & Reference & & Reference & \\
\hline Senior $(n=438)$ & $65.5(7.5)$ & $0.92(0.01,1.84)$ & & $1.00(0.07,1.92)$ & \\
\hline Tertiary $(n=109)$ & $66.8(8.0)$ & $2.14(0.65,3.64)$ & 0.023 & $2.08(0.57,3.59)$ & 0.022 \\
\hline \multicolumn{6}{|l|}{ Marital status } \\
\hline Single $(n=116)$ & $64.2(6.4)$ & $-1.19(-2.59,0.21)$ & & $-1.26(-2.69,0.16)$ & \\
\hline Married/cohabiting $(n=967)$ & $65.4(7.4)$ & Reference & & Reference & \\
\hline Separated/widowed $(n=35)$ & $63.5(6.0)$ & $-1.91(-4.36,0.54)$ & 0.089 & $-1.91(-4.38,0.54)$ & 0.075 \\
\hline \multicolumn{6}{|l|}{ Area of residence } \\
\hline Urban $(n=770)$ & $65.3(7.5)$ & Reference & & & \\
\hline Rural $(n=336)$ & $64.9(6.8)$ & $0.49(-1.42,0.44)$ & 0.302 & & \\
\hline \multicolumn{6}{|l|}{ Alcohol use } \\
\hline No $(n=775)$ & $65.3(7.5)$ & Reference & & & \\
\hline Yes $(n=343)$ & $65.0(6.6)$ & $-0.34(-1.26,0.59)$ & 0.477 & & \\
\hline \multicolumn{6}{|l|}{ Infections } \\
\hline \multicolumn{6}{|l|}{ HIV } \\
\hline Uninfected $(n=1002)$ & $65.2(7.3)$ & Reference & & & \\
\hline Infected $(n=117)$ & $64.9(6.5)$ & $-0.35(-1.74,1.05)$ & 0.626 & & \\
\hline \multicolumn{6}{|l|}{ Asymptomatic malaria } \\
\hline Uninfected $(n=991)$ & $65.2(7.4)$ & Reference & & & \\
\hline Infected $(n=109)$ & $64.9(6.6)$ & $-0.29(-1.73,1.15)$ & 0.695 & & \\
\hline \multicolumn{6}{|l|}{ Schistosoma mansoni } \\
\hline Uninfected $(n=908)$ & $65.2(7.1)$ & Reference & & & \\
\hline Infected $(n=204)$ & $65.5(7.7)$ & $0.31(-0.79,1.41)$ & 0.579 & & \\
\hline \multicolumn{6}{|l|}{ Hookworm (Necator americanus) } \\
\hline Uninfected $(n=662)$ & $65.1(7.1)$ & Reference & & & \\
\hline Infected $(n=450)$ & $65.4(7.4)$ & $0.27(-0.60,1.14)$ & 0.539 & & \\
\hline \multicolumn{6}{|l|}{ Ascaris lumbricoides } \\
\hline Uninfected $(n=1084)$ & $65.3(7.3)$ & Reference & & & \\
\hline Infected $(n=28)$ & $65.1(5.5)$ & $-0.18(-2.90,2.54)$ & 0.896 & & \\
\hline \multicolumn{6}{|l|}{ Intervention one } \\
\hline Placebo $(n=566)$ & $65.0(6.9)$ & Reference & & & \\
\hline Albendazole $(n=553)$ & $65.4(7.7)$ & $0.39(-0.46,1.24)$ & 0.366 & & \\
\hline \multicolumn{6}{|l|}{ Intervention two } \\
\hline Placebo $(n=564)$ & $65.4(7.3)$ & Reference & & & \\
\hline Praziquantel $(n=555)$ & $65.0(7.2)$ & $-0.44(-1.29,0.42)$ & 0.315 & & \\
\hline \multicolumn{6}{|l|}{ Level 2: Factors in childhood } \\
\hline Birth weight $(\mathrm{kg})(n=932)$ & & $0.66(-0.27,1.59)$ & 0.164 & $0.57(-0.40,1.53)$ & 0.246 \\
\hline \multicolumn{6}{|l|}{ Sex } \\
\hline Male $(n=583)$ & $64.9(7.2)$ & Reference & & Reference & \\
\hline Female $(n=536)$ & $65.5(7.4)$ & $0.64(-0.21,1.49)$ & 0.141 & $0.49(-0.43,1.42)$ & 0.294 \\
\hline Sickle-cell trait & & & & & \\
\hline $\operatorname{HbAA}(n=661)$ & $65.4(7.1)$ & Reference & & & \\
\hline $\operatorname{HbAS}(n=141)$ & $65.5(7.4)$ & $0.15(-1.16,1.46)$ & 0.825 & & \\
\hline Season of birth & & & & & \\
\hline Dry $(n=651)$ & $65.5(7.3)$ & Reference & & Reference & \\
\hline Wet $(n=468)$ & $64.7(7.2)$ & $-0.79(-1.65,0.07)$ & 0.073 & $0.59(-1.52,0.35)$ & 0.214 \\
\hline Place of delivery & & & & & \\
\hline Entebbe Hospital $(n=824)$ & $65.1(7.1)$ & Reference & & & \\
\hline Home $(n=120)$ & $65.4(8.5)$ & $0.36(-1.03,1.76)$ & & & \\
\hline Others $(n=174)$ & $65.7(7.3)$ & $0.61(-0.58,1.80)$ & 0.564 & & \\
\hline Feeding status (at 6 week of age) & & & & & \\
\hline Exclusive breast fed $(n=748)$ & $65.4(7.4)$ & Reference & & & \\
\hline Mixed fed $(n=344)$ & $64.7(7.0)$ & $-0.63(-1.56,0.30)$ & & & \\
\hline Weaned $(n=14)$ & $67.1(4.4)$ & $1.78(-2.07,5.63)$ & 0.251 & & \\
\hline
\end{tabular}


Table 2 (continued)

\begin{tabular}{|c|c|c|c|c|c|}
\hline Factors & Mean BP (SD) & Crude $\beta(95 \% \mathrm{CI})$ & $P$-value & Adjusted $\beta(95 \% \mathrm{CI})$ & $P$-value \\
\hline \multicolumn{6}{|l|}{ Intervention three } \\
\hline Placebo $(n=553)$ & $64.9(7.0)$ & Reference & & Reference & \\
\hline Albendazole $(n=554)$ & $65.5(7.5)$ & $0.62(-0.24,1.47)$ & 0.156 & $0.56(-0.37,1.48)$ & 0.233 \\
\hline \multicolumn{6}{|l|}{ HIV status } \\
\hline Unexposed $(n=1001)$ & $65.2(7.3)$ & Reference & & & \\
\hline Exposed not infected $(n=100)$ & $65.1(6.7)$ & $-0.12(-1.62,1.37)$ & & & \\
\hline Infected $(n=18)$ & $63.5(5.1)$ & $-1.71(-5.10,1.68)$ & 0.609 & & \\
\hline \multicolumn{6}{|l|}{ Malaria infection below 5 years of age } \\
\hline \multicolumn{6}{|l|}{ Clinical or asymptomatic malaria ${ }^{\mathrm{a}}$} \\
\hline No $(n=456)$ & $65.9(7.1)$ & Reference & & Reference & \\
\hline Yes $(n=663)$ & $64.6(7.3)$ & $-1.28(-2.14,-0.41)$ & 0.004 & $-1.47(-2.41,-0.53)$ & 0.002 \\
\hline \multicolumn{6}{|l|}{ Clinical malaria $^{\mathrm{a}}$} \\
\hline None $(n=474)$ & $66.0(7.2)$ & Reference & & Reference & \\
\hline Yes $(n=645)$ & $64.6(7.3)$ & $-1.38(-2.24,-0.51)$ & 0.002 & $-1.33(-2.26,-0.39)$ & 0.005 \\
\hline \multicolumn{6}{|l|}{ Episodes of clinical malaria ${ }^{a}$} \\
\hline None $(n=474)$ & $65.9(7.2)$ & Reference & & Reference & \\
\hline $1-2(n=382)$ & $64.5(7.3)$ & $-1.45(-2.42,-0.47)$ & & $-1.53(-2.59,-0.46)$ & \\
\hline$\geq 3(n=263)$ & $64.9(7.4)$ & $-1.02(-2.12,0.07)$ & 0.011 & $-1.03(-2.22,0.16)$ & 0.015 \\
\hline \multicolumn{6}{|l|}{ Asymptomatic malaria ${ }^{a}$} \\
\hline None $(n=983)$ & $64.5(7.3)$ & Reference & & Reference & \\
\hline Yes $(n=124)$ & $64.9(7.4)$ & $-1.45(-2.80,-0.10)$ & 0.035 & $-1.35(-2.89,0.18)$ & 0.082 \\
\hline \multicolumn{6}{|l|}{ Schistosoma mansoni } \\
\hline Uninfected $(n=1076)$ & $65.2(7.3)$ & Reference & & & \\
\hline Infected $(n=33)$ & $64.5(5.8)$ & $0.67(-3.18,1.84)$ & 0.602 & & \\
\hline \multicolumn{6}{|l|}{ Ascaris lumbricoides } \\
\hline Uninfected $(n=1052)$ & $65.2(7.3)$ & Reference & & & \\
\hline Infected $(n=57)$ & $64.5(7.1)$ & $-0.75(-2.68,1.18)$ & 0.445 & & \\
\hline \multicolumn{6}{|l|}{ Hookworm (Necator americanus) } \\
\hline Uninfected $(n=1085)$ & $65.2(7.3)$ & Reference & & Reference & \\
\hline Infected $(n=24)$ & $62.9(5.8)$ & $-2.29(-5.22,0.64)$ & 0.125 & $-1.79(-4.93,1.35)$ & 0.261 \\
\hline \multicolumn{6}{|l|}{ Trichuris trichiura } \\
\hline Uninfected $(n=997)$ & $65.1(7.2)$ & Reference & & & \\
\hline Infected $(n=112)$ & $65.8(7.7)$ & $0.67(-0.74,2.09)$ & 0.353 & & \\
\hline \multicolumn{6}{|l|}{ Microfilaria (Mansonella perstans) } \\
\hline Uninfected $(n=1102)$ & $65.1(7.2)$ & Reference & & & \\
\hline Infected $(n=8)$ & $67.3(3.3)$ & $2.12(-2.91,7.14)$ & 0.409 & & \\
\hline \multicolumn{6}{|l|}{ Level 3: Factors in adolescence } \\
\hline Age (years) & & $1.85(1.00,2.70)$ & $<0.001$ & $1.53(0.63,2.43)$ & $<0.001$ \\
\hline Body mass index $\left(\mathrm{kg} / \mathrm{m}^{2}\right)$ & & $0.28(0.20,0.36)$ & $<0.001$ & $0.74(0.42,1.05)$ & $<0.001$ \\
\hline Waist circumference $(\mathrm{cm})$ & & $0.88(0.66,1.10)$ & $<0.001$ & $0.07(-0.05,0.18)$ & 0.279 \\
\hline \multicolumn{6}{|l|}{ Family history } \\
\hline \multicolumn{6}{|l|}{ High blood pressure } \\
\hline No $(n=1000)$ & $65.0(7.2)$ & Reference & & Reference & \\
\hline Yes $(n=105)$ & $66.7(7.6)$ & $1.65(0.19,3.12)$ & 0.027 & $1.57(0.08,3.06)$ & 0.037 \\
\hline Diabetes & & & & & \\
\hline No $(n=927)$ & $65.2(7.2)$ & Reference & & & \\
\hline Yes $(n=186)$ & $65.5(7.8)$ & $0.35(-0.80,1.49)$ & 0.553 & & \\
\hline Body composition analysis ${ }^{\mathrm{c}}$ & & & & & \\
\hline Fat mass index ${ }^{\mathrm{b}}\left(\mathrm{kg} / \mathrm{m}^{2}\right)(n=176)$ & & $1.75(0.83,2.69)$ & $<0.001$ & $0.87(-0.73,2.47)$ & 0.255 \\
\hline Fat-free mass index $\mathrm{x}^{\mathrm{b}}\left(\mathrm{kg} / \mathrm{m}^{2}\right)(n=176)$ & & $1.19(0.40,1.98)$ & 0.003 & $0.28(-0.90,1.45)$ & 0.622 \\
\hline Total body water index $\left(\mathrm{kg} / \mathrm{m}^{2}\right)(n=176)$ & & $2.13(0.95,3.30)$ & $<0.001$ & $1.51(-0.86,3.88)$ & 0.180 \\
\hline Adding salt to cooked food at the table & & & & & \\
\hline No $(n=20)$ & $67.4(6.1)$ & $2.19(-1.04,5.41)$ & & $2.72(-0.39,5.82)$ & \\
\hline Yes $(n=1086)$ & $65.2(7.3)$ & Reference & 0.184 & Reference & 0.083 \\
\hline Days a fruit is eaten/week & & & & & \\
\hline $0-2(n=543)$ & $65.7(7.1)$ & Reference & & Reference & \\
\hline $3-7(n=541)$ & $64.7(7.5)$ & $-0.98(-1.85,-0.11)$ & 0.028 & $-0.96(-1.83,-0.10)$ & 0.027 \\
\hline Days vegetables eaten/week & & & & & \\
\hline $0-2(n=461)$ & $65.4(7.1)$ & Reference & & & \\
\hline $3-7(n=635)$ & $65.1(7.5)$ & $-0.27(-1.15,0.60)$ & 0.540 & & \\
\hline Days animal-protein eaten/week & & & & & \\
\hline $0-2(n=726)$ & $65.1(6.9)$ & Reference & & & \\
\hline
\end{tabular}


Table 2 (continued)

\begin{tabular}{|c|c|c|c|c|c|}
\hline Factors & Mean BP (SD) & Crude $\beta(95 \% \mathrm{CI})$ & $P$-value & Adjusted $\beta(95 \% \mathrm{CI})$ & $P$-value \\
\hline $3-7(n=374)$ & $65.4(8.0)$ & $0.30(-0.61,1.20)$ & 0.523 & & \\
\hline \multicolumn{6}{|l|}{ Days sugared drinks taken/week } \\
\hline None $(n=427)$ & $65.0(7.1)$ & Reference & & Reference & \\
\hline $1-3(n=492)$ & $65.2(7.4)$ & $0.25(-0.70,1.20)$ & & $0.12(-0.84,1.08)$ & \\
\hline $4-7(n=174)$ & $66.0(7.5)$ & $1.06(-0.23,2.35)$ & 0.271 & $0.54(-0.75,1.83)$ & 0.707 \\
\hline \multicolumn{6}{|l|}{ Days a fruit is eaten/week } \\
\hline Days vegetables eaten/week & & $0.02(-0.16,0.1)$ & 0.800 & & \\
\hline Days animal-protein eaten/week & & $0.14(-0.11,0.39)$ & 0.284 & & \\
\hline Days starchy foods eaten/week & & $0.03(-0.50,0.55)$ & 0.924 & & \\
\hline Days sugared drinks taken/week & & $0.20(0.00,0.41)$ & 0.048 & & \\
\hline \multicolumn{6}{|l|}{ Breast development (girls only) ${ }^{\mathrm{b}}$} \\
\hline Pre-pubertal $(n=178)$ & $64.1(6.1)$ & Reference & & Reference & \\
\hline Pubertal $(n=97)$ & $67.2(7.9)$ & $3.067(1.38,4.76)$ & $<0.001$ & $0.98(-0.88,2.84)$ & 0.281 \\
\hline \multicolumn{6}{|l|}{ Pubic hair development ${ }^{\mathrm{b}}$} \\
\hline Pre-pubertal $(n=441)$ & $64.1(6.6)$ & Reference & & Reference & \\
\hline Pubertal $(n=170)$ & $66.1(7.6)$ & $2.04(0.82,3.26)$ & 0.001 & $0.68(-0.62,1.99)$ & 0.293 \\
\hline \multicolumn{6}{|l|}{ Snoring } \\
\hline No $(n=932)$ & $65.1(7.2)$ & Reference & & & \\
\hline Yes $(n=163)$ & $65.6(7.8)$ & $0.44(-0.78,1.66)$ & 0.477 & & \\
\hline \multicolumn{6}{|l|}{ Duration of night sleep } \\
\hline$<9$ hours $(n=306)$ & $65.8(7.6)$ & Reference & & Reference & \\
\hline 9 hours $(n=382)$ & $64.8(7.1)$ & $-1.03(-2.11,0.06)$ & & $-0.92(-2.02,0.18)$ & \\
\hline$>9$ hours $(n=405)$ & $65.2(7.2)$ & $-0.79(-1.86,0.28)$ & 0.160 & $-0.67(-1.76,0.43)$ & 0.240 \\
\hline \multicolumn{6}{|l|}{ Smoking in household } \\
\hline Non $(n=962)$ & $65.2(7.3)$ & Reference & & & \\
\hline Yes $(n=147)$ & $65.0(6.8)$ & $-0.21(-1.46,1.06)$ & 0.745 & & \\
\hline \multicolumn{6}{|l|}{ Type of school attended } \\
\hline Day $(n=117)$ & $65.1(7.2)$ & Reference & & Reference & \\
\hline Boarding school $(n=719)$ & $66.2(7.8)$ & $1.13(-0.26,2.52)$ & 0.112 & $-0.24(-1.67,1.20)$ & 0.737 \\
\hline \multicolumn{6}{|c|}{ Involved in physical education at school } \\
\hline No $(n=385)$ & $65.0(6.9)$ & Reference & & & \\
\hline Yes $(n=719)$ & $65.3(7.5)$ & $0.32(-0.58,1.22)$ & 0.482 & & \\
\hline \multicolumn{6}{|c|}{ Infections at the time of blood pressure measurement } \\
\hline \multicolumn{6}{|c|}{ Asymptomatic malaria } \\
\hline Uninfected $(n=1067)$ & $65.3(7.3)$ & Reference & & & \\
\hline Infected $(n=22)$ & $64.0(5.5)$ & $-1.31(-4.36,1.75)$ & 0.401 & & \\
\hline \multicolumn{6}{|l|}{ Schistosoma mansoni } \\
\hline Uninfected $(n=964)$ & $65.2(7.4)$ & Reference & & & \\
\hline Infected $(n=112)$ & $65.0(5.8)$ & $-0.19(-1.62,1.24)$ & 0.791 & & \\
\hline \multicolumn{6}{|c|}{ Hookworm (Necator americanus) } \\
\hline Uninfected $(n=1066)$ & $65.2(7.3)$ & Reference & & & \\
\hline Infected $(n=10)$ & $64.0(5.9)$ & $-1.25(-5.80,3.30)$ & 0.590 & & \\
\hline \multicolumn{6}{|l|}{ Ascaris (Ascaris lumbricoides) } \\
\hline Uninfected $(n=1073)$ & $65.2(7.3)$ & Reference & & & \\
\hline Infected $(n=3)$ & $62.3(4.3)$ & $-2.86(-11.14,5.42)$ & 0.498 & & \\
\hline \multicolumn{6}{|l|}{ Trichuris trichiura } \\
\hline Uninfected $(n=1036)$ & $65.1(7.2)$ & Reference & & & \\
\hline Infected $(n=40)$ & $66.4(9.4)$ & $1.23(-1.07,3.54)$ & 0.294 & & \\
\hline
\end{tabular}

Model building followed the hierarchical approach, adding factors sequentially at three levels starting with the distal factors (level 1). Factors at the same level were added to the model at the same time and considered confounders for each other and for proximal factors. A $P$-value $<0.20$ was used for considering the inclusion and maintenance of factors in the model

Adjusted $\beta$ for which $95 \%$ CI exclude 0 are highlighted in bold

$\beta$ linear regression coefficient: mean difference in blood pressure (BP) measured in $\mathrm{mmHg}$

${ }^{a}$ Not included in the model together but each was adjusted for all other variables in the model

${ }^{b}$ Not included in multivariable model building for other exposures because of large proportion of missing information; but each was adjusted for variables in the final model building

${ }^{\mathrm{c}}$ Not adjusted for body mass index because body mass index is on the causal pathway 
with only $22(2.1 \%)$ adolescents had parasitaemic at the time of BP measurement.

Sub-microscopic malaria was most likely misclassified as negative in this population, since in malaria-endemic areas, asymptomatic malaria often presents as sub-microscopic in individuals with past malaria infection [27]. We found no association between sickle-cell trait and adolescent BP; contrary to the hypothesis advanced by Etyang et al., who used sickle-cell trait as an instrumental variable in a Mendelian randomisation study [28]. In the predominantly adult populations from Kenya, sickle-cell trait (linked with partial protection against malaria) was associated with lower BP in Kilifi (currently a low-moderate but historically a high malaria transmission area) compared to Nairobi (no malaria transmission) [29]. The differences in malaria exposure intensity and participant age distribution between our study and the Kenyan study could explain our contrasting results.

Similar to earlier studies [30, 31], childhood malaria was associated with reductions in both weight and height, and some of the inverse association seen in this study may be explained by this mechanism, or by confounding by unmeasured factors. The escalating burden of high BP has coincided with the declining malaria burden on the African continent $[2,32,33]$. This could be explained by the epidemiological transition process on continent, or the effect could be more direct; the mechanisms remain to be elucidated.

Current but not previous infection with Trichuris trichiura (a type of soil transmitted helminth, commonly known as whipworm) was associated with increased systolic BP in early adolescence. To our knowledge, no study has previously reported such an association. This may reflect short-term effects (probably arterial stiffness from inflammatory reaction) or it could be a spurious finding due to the many exposures included in the analysis. The effect of current Trichuris trichiura infection on BP is likely not mediated through increasing BMI (weight or height); there was no difference in these measures between adolescents with and without current Trichuris trichiura infection.

Unlike previous studies [34], we found no association between BP and salt intake. The lack of evidence for this relationship in our study could be due to measurement error from self-report, or the fact that nearly everyone added salt to cooked food. Measuring sodium in a 24-h urine sample or in commonly consumed local foods would provide a more accurate reflection of daily intake. Physical activity was not associated with lower BP, contrary to earlier literature [35]; sedentary lifestyles are still fairly uncommon in this population.

Previous studies have linked hypertension to socioeconomic determinants (socioeconomic status (SES), education, income, urbanisation) $[12,36]$. Our study is consistent with a Uganda study in adults which showed that
BP was not associated with urban residence [37] but contrary to studies linking increased BP with low SES [36] and urbanisation [12]. We have shown that higher maternal education was associated with increased BP in adolescents, whereas other studies, predominantly from high-income countries, report an inverse association [36]. Although low SES and education is associated with hypertension in the developed world [36], the relationship may be inverse in less developed countries [38]. In these settings, offspring from more highly educated households are more likely to have sedentary lifestyles and unhealthy dietary practices, and to be obese, compared to offspring from less-educated households.

Strengths of this study included its longitudinal design with prospectively collected data reducing recall and reporter bias, the use of robust $\mathrm{BP}$ procedures and the measurement of BP on up to two extra occasions in those with $\mathrm{BP} \geq 95$ th percentile at the initial visit, to avoid overestimation of high BP. It is unlikely that white-coat phenomenon was an issue as participants regularly attend this clinic for scheduled and/or illness visits. The use of digital machines reduced differences in BP reading between operators which can occur with auscultation.

Study limitations include the possibility of residual confounding by unmeasured factors (such as glomerular filtration rate (GFR)). The GFR could not be estimated as creatinine was only measured for a subgroup of the participants. The use of digital BP machines may overestimate BP; however, digital devices used in this study were calibrated twice annually. A large number of statistical tests were undertaken; thus, some findings may be due to multiplicity. However, it is reassuring that most findings are consistent with previous literature, albeit from different settings. Not inviting all adolescents (those with pre-hypertension or normal BP on day 1) for up to two extra BP measurements might have resulted in an underestimation in the overall prevalence of pre-hypertension and hypertension. We modelled BP as a continuous outcome, since analysing high or pre-hypertensive BP versus normal BP as a binary outcome (or outcomes) would be underpowered, consequently our findings may not necessarily reflect associations with hypertensive disease.

In summary, routine BP screening which is seldom conducted for adolescents at health care visits remains vital in the control and prevention of CVDs later in life. Similar life-course factors to those observed in high-income settings (such as adiposity and diet) affect both systolic BP and diastolic BP among African adolescents. Interventions during pregnancy, childhood and early adolescence could be vital in the control and prevention of later high BP. Multiple intervention strategies initiated during pregnancy and the early postnatal period and continued across a lifetime could be fundamental in the control of adulthood hypertension and CVDs. 


\section{Summary}

\section{What is known about the topic}

- High blood pressure and cardiovascular diseases are increasing in Africa.

- Scarcity of data on blood pressure risk factors among African children and adolescents.

- The risk factors for high blood pressure may differ from those seen in high-income non-tropical settings.

\section{What this paper adds}

- Malaria infection in childhood is associated with reduced blood pressure among adolescents. Effects of childhood malaria on later blood pressure may be partially mediated through chronic reduction in weight and height.

- Current infection with Trichuris trichiura is associated with increased blood pressure.

- Interventions during pregnancy, childhood and early adolescence could be vital in the prevention of high blood pressure later in life.

Acknowledgements We thank all staff, participants and parents/ guardians in the EMaBS, Entebbe Hospital midwives, the community field teams (Entebbe and Katabi), MRC/UVRI \& LSHTM Uganda Research Unit staff, and Mulago Hospital staff. We thank all individuals involved in the generation and curation of the genotype and imputed data including Adrian VS Hill, Manjinder Sandhu, Deept Gurdasani, Tommy Carstensen, Allan Muhwezi, Beatrice Nassanga and staff at the Wellcome Sanger Institute and Wellcome Centre for Human Genetics.

Funding This work was supported by: the Wellcome Trust (Grant Numbers: 064693, 079110, 95778, to Alison M. Elliott; 106289/Z/14/ Z, to Liam Smeeth; and 098504/Z/12/Z, to Alexander Mentzer); UK Medical Research Council and UK Department for International Development (Grant Number: MR/K012126/1, to Emily L. Webb); and Commonwealth Scholarship Commission (Grant Number: UGCS2015-808, to Swaib A. Lule).

\section{Compliance with ethical standards}

Conflict of interest The authors declare that they have no conflict of interest.

Publisher's note: Springer Nature remains neutral with regard to jurisdictional claims in published maps and institutional affiliations.

Open Access This article is licensed under a Creative Commons Attribution 4.0 International License, which permits use, sharing, adaptation, distribution and reproduction in any medium or format, as long as you give appropriate credit to the original author(s) and the source, provide a link to the Creative Commons license, and indicate if changes were made. The images or other third party material in this article are included in the article's Creative Commons license, unless indicated otherwise in a credit line to the material. If material is not included in the article's Creative Commons license and your intended use is not permitted by statutory regulation or exceeds the permitted use, you will need to obtain permission directly from the copyright holder. To view a copy of this license, visit http://creativecommons. org/licenses/by/4.0/.

\section{References}

1. Donnison CP. Pressure in the African Native: its bearing upon the aetiology of hyperpiesia and arteriosclerosis. Lancet. 1929;213:6-7.

2. Dalal S, Beunza JJ, Volmink J, Adebamowo C, Bajunirwe F, Njelekela $\mathrm{M}$, et al. Non-communicable diseases in sub-Saharan Africa: what we know now. Int J Epidemiol. 2011;40:885-901.

3. Lopez AD, Mathers CD, Ezzati M, Jamison DT, Murray CJ. Global and regional burden of disease and risk factors, 2001: systematic analysis of population health data. Lancet. 2006;367:1747-57.

4. Kagura J, Adair LS, Musa MG, Pettifor JM, Norris SA. Blood pressure tracking in urban black South African children: birth to twenty cohort. BMC Pediatr. 2015;15:78.

5. National High Blood Pressure Education Program Working Group on High Blood Pressure in Children and Adolescents. The fourth report on the diagnosis, evaluation, and treatment of high blood pressure in children and adolescents. Pediatrics. 2004;114(2 Suppl, 4th Report):555-76.

6. Hardy R, Lawlor DA, Kuh D. A life course approach to cardiovascular aging. Future Cardiol. 2015;11:101-13.

7. Din-Dzietham R, Liu Y, Bielo MV, Shamsa F. High blood pressure trends in children and adolescents in national surveys, 1963 to 2002. Circulation. 2007;116:1488-96.

8. Noubiap JJ, Essouma M, Bigna JJ, Jingi AM, Aminde LN, Nansseu JR. Prevalence of elevated blood pressure in children and adolescents in Africa: a systematic review and meta-analysis. Lancet Public Health. 2017;2:e375-e86.

9. Gill DG, Mendes de Costa B, Cameron JS, Joseph MC, Ogg CS, Chantler C. Analysis of 100 children with severe and persistent hypertension. Arch Dis Child. 1976;51:951-6.

10. Falkner B, Daniels SR. Summary of the fourth report on the diagnosis, evaluation, and treatment of high blood pressure in children and adolescents. Hypertension. 2004;44:387-8.

11. Hansen ML, Gunn PW, Kaelber DC. Underdiagnosis of hypertension in children and adolescents. JAMA. 2007;298:874-9.

12. Addo J, Smeeth L, Leon DA. Hypertension in sub-saharan Africa: a systematic review. Hypertension. 2007;50:1012-8.

13. Jobe M, Agbla SC, Prentice AM, Hennig BJ. High blood pressure and associated risk factors as indicator of preclinical hypertension in rural West Africa: A focus on children and adolescents in The Gambia. Medicine. 2017;96:e6170.

14. Afrifa-Anane E, Agyemang C, Codjoe SN, Ogedegbe G, de-Graft Aikins A. The association of physical activity, body mass index and the blood pressure levels among urban poor youth in Accra, Ghana. BMC Public Health. 2015;15:269.

15. Elliott AM, Kizza M, Quigley MA, Ndibazza J, Nampijja M, Muhangi L, et al. The impact of helminths on the response to immunization and on the incidence of infection and disease in childhood in Uganda: design of a randomized, double-blind, placebo-controlled, factorial trial of deworming interventions delivered in pregnancy and early childhood [ISRCTN32849447]. Clin Trials. 2007;4:42-57.

16. Ndibazza J, Webb EL, Lule S, Mpairwe H, Akello M, Oduru G, et al. Associations between maternal helminth and malaria 
infections in pregnancy and clinical malaria in the offspring: a birth cohort in entebbe, Uganda. J Infect Dis. 2013;208: 2007-16.

17. Lule SA, Namara B, Akurut H, Muhangi L, Lubyayi L, Nampijja $\mathrm{M}$, et al. Are birth weight and postnatal weight gain in childhood associated with blood pressure in early adolescence? Results from a Ugandan birth cohort. Int J Epidemiol. 2019;48:148-56.

18. Marshall WA, Tanner JM. Variations in pattern of pubertal changes in girls. Arch Dis Child. 1969;44:291-303.

19. Gurdasani D, Carstensen T, Tekola-Ayele F, Pagani L, Tachmazidou I, Hatzikotoulas K, et al. The African Genome Variation Project shapes medical genetics in Africa. Nature. 2015;517: 327-32.

20. Katz N, Chaves A, Pellegrino J. A simple device for quantitative stool thick-smear technique in Schistosomiasis mansoni. Rev Inst Med Trop Sao Paulo. 1972;14:397-400.

21. Webb EL, Mawa PA, Ndibazza J, Kizito D, Namatovu A, Kyosiimire-Lugemwa J, et al. Effect of single-dose anthelmintic treatment during pregnancy on an infant's response to immunisation and on susceptibility to infectious diseases in infancy: a randomised, double-blind, placebo-controlled trial. Lancet. 2011;377:52-62.

22. Melrose WD, Turner PF, Pisters P, Turner B. An improved Knott's concentration test for the detection of microfilariae. Trans R Soc Trop Med Hyg. 2000;94:176.

23. Muhangi L, Woodburn P, Omara M, Omoding N, Kizito D, Mpairwe $\mathrm{H}$, et al. Associations between mild-to-moderate anaemia in pregnancy and helminth, malaria and HIV infection in Entebbe, Uganda. Trans R Soc Trop Med Hyg. 2007;101:899-907.

24. Woodburn PW, Muhangi L, Hillier S, Ndibazza J, Namujju PB, Kizza M, et al. Risk factors for helminth, malaria, and HIV infection in pregnancy in Entebbe, Uganda. PLoS Negl Trop Dis. 2009;3:e473.

25. Kidy F, Rutebarika D, Lule SA, Kizza M, Odiit A, Webb EL, et al. Blood pressure in primary school children in Uganda: a cross-sectional survey. BMC Public Health. 2014;14:1223.

26. Pienovi L, Lara M, Bustos P, Amigo H. Fruit and vegetable intake, and blood pressure. A population research. Arch Latinoam Nutr. 2015;65:21-6.
27. Lin JT, Saunders DL, Meshnick SR. The role of submicroscopic parasitemia in malaria transmission: what is the evidence? Trends Parasitol. 2014;30:183-90.

28. Etyang AOS, Cruickshank L, Scott JK, New JAG. hypotheses in clinical medicine: the malaria-high blood pressure hypothesis. Circ Res. 2016;119:36-40.

29. Etyang AO. Determining the causal role of malaria in elevating blood pressure and pulse wave velocity in kenyan adolescents and adults. Doctoral Thesis, London School of Hygiene \& Tropical Medicine; 2017.

30. ter Kuile FO, Terlouw DJ, Kariuki SK, Phillips-Howard PA, Mirel LB, Hawley WA, et al. Impact of permethrin-treated bed nets on malaria, anemia, and growth in infants in an area of intense perennial malaria transmission in western Kenya. Am J Trop Med Hyg. 2003;68(4 Suppl):68-77.

31. Bradley-Moore AM, Greenwood BM, Bradley AK, Kirkwood BR, Gilles HM. Malaria chemoprophylaxis with chloroquine in young Nigerian children. III. Its Eff Nutr Ann Trop Med Parasitol. 1985;79:575-84.

32. Bhatt S, Weiss DJ, Cameron E, Bisanzio D, Mappin B, Dalrymple $\mathrm{U}$, et al. The effect of malaria control on Plasmodium falciparum in Africa between 2000 and 2015. Nature. 2015;526:207-11.

33. Noor AM, Kinyoki DK, Mundia CW, Kabaria CW, Mutua JW, Alegana VA, et al. The changing risk of Plasmodium falciparum malaria infection in Africa: 2000-10: a spatial and temporal analysis of transmission intensity. Lancet. 2014;383:1739-47.

34. Brown IJ, Tzoulaki I, Candeias V, Elliott P. Salt intakes around the world: implications for public health. Int $\mathrm{J}$ Epidemiol. 2009;38:791-813.

35. Diaz KM, Shimbo D. Physical activity and the prevention of hypertension. Curr Hypertens Rep. 2013;15:659-68.

36. Leng B, Jin Y, Li G, Chen L, Jin N. Socioeconomic status and hypertension: a meta-analysis. J Hypertens. 2015;33:221-9.

37. Guwatudde D, Mutungi G, Wesonga R, Kajjura R, Kasule H, Muwonge J, et al. The epidemiology of hypertension in Uganda: findings from the national non-communicable diseases risk factor survey. PLoS ONE. 2015;10:e0138991.

38. Caballero B. The global epidemic of obesity: an overview. Epidemiol Rev. 2007;29:1-5. 